\title{
Liver metastases in gastroenteropancreatic neuroendocrine tumours - treatment methods
}

\author{
Paweł Gut \\ Department of Endocrinology, Metabolism and Internal Medicine, Poznan University of Medical Sciences, Poznan, Poland \\ Gastroenterology Rev 2020; 15 (3): 207-214 \\ DOI: https://doi.org/10.5114/pg.2020.91501
}

Key words: gastroenteropancreatic neuroendocrine tumours, liver metastases.

Address for correspondence: Paweł Gut MD, PhD, Department of Endocrinology, Metabolism and Internal Medicine, Poznan University of Medical Sciences, 49 Przybyszewskiego St, 60-355 Poznan, Poland, phone: +48 618691 322, e-mail: gutpj@poczta.onet.pl

\begin{abstract}
Surgical approaches that allow the safe treatment of multiple, bilateral, large tumours, and that combine extirpative, ablative and interventional therapies, have expanded the population of patients with neuroendocrine tumors (NET) liver metastases (LMs) who can benefit from aggressive treatment of their liver disease. Pre-treatment staging often includes the biochemical assessment of serologic markers such as serotonin, insulin, vasoactive intestinal peptide, and chromogranin, even in patients without clinically apparent hormonal excess. Radiofrequency ablation (RFA) is a technique that involves the use of thermal energy to induce coagulation necrosis, thereby destroying tumour cells. Resection plus RFA is increasingly used in patients with bilateral NET LMs. Resection is performed for large or dominant lesions, while ablation is used to treat small lesions. Hepatic arterial embolization, typically termed transarterial embolization, and transarterial chemoembolization have been shown to induce a reduction in tumour size and to ameliorate symptoms of excess hormonal secretion.
\end{abstract}

\section{Introduction}

Surgical management for gastroenteropancreatic neuroendocrine tumours (GEP-NETs) metastatic to the liver has evolved in parallel with advances in surgical therapy for liver tumours, advances in nonsurgical therapies for neuroendocrine tumours (NETs), and a better understanding of the variable biology of NETs. Surgical approaches that allow the safe treatment of multiple, bilateral, large tumours, and that combine extirpative, ablative and interventional therapies, have expanded the population of patients with NET liver metastases (LMs) who can benefit from aggressive treatment of their liver disease. Recognition that the safety of extensive resection is related to the future liver remnant (FLR), or liver that will remain after resection, has led to a shift in focus from what is resected to what remains after resection. Methods such as preoperative portal vein embolization (PVE), designed to increase the volume and function of the FLR before surgery, help to minimize the risk of liver insufficiency and failure, and shift the landscape of major resection from high risk to a much safer alternative for patients with a more extensive liver metastatic burden. Methods which help to ensure efficient recovery and long survival after hepatectomy for metastasis are mandatory for patients who may have very prolonged survival following optimal treatment. Integrated medical, interventional and surgical therapies are needed due to the unfortunate reality that the majority of patients with NET LMs have unresectable disease. Several factors converge to focus interest in surgical therapy for NET LMs. First, up to $90 \%$ of patients with NETs will develop LMs, and up to $40 \%$ of patients with NETs are found to have LMs at the time of diagnosis [1]. Moreover, LMs are thought to determine patient survival in patients with NETs [2]. Specifically, the 5-year overall survival rate for patients with midgut NETs (carcinoids) without LMs ranges from $75 \%$ to $99 \%$, whereas the 5 -year survival rate among NET patients with LMs is only $0-40 \%[3,4]$. A similar trend is seen for patients with foregut NETs such as gastrinoma, with a reported 10 -year overall survival of $98 \%$ without LMs vs. $85 \%$ in those with metachronous LMs, and $26 \%$ in those with LMs at the time of diagnosis of the primary gastrinoma [5]. Remarkably, however, a significant proportion of patients with metastatic NETs exhibit indolent biological behaviour [6], although recurrence after an extended disease-free interval is common, and 
recurrences may progress more rapidly than the previously indolent disease. These factors entail the need for careful follow-up and for a measured, integrated approach to medical and surgical treatment. An additional dimension to the treatment of patients with NETs is the presence or absence of hormonal excess syndromes. Many patients with NETs have non-functional tumours, or tumours which produce hormones that lack any clinically significant effect. Others have functional tumours and may suffer from a wide range of hormonal excess syndromes, resulting in dermatologic, endocrine, gastrointestinal and cardiovascular clinical effects. Generally, patients with midgut NETs without LMs do not exhibit symptoms of hormonal excess, despite tumours which secrete functional hormones into the portal circulation, as these hormones pass directly into the liver, which can metabolize them to an inactive form on the first pass, preventing their systemic circulation and effects. In contrast, functional NET LMs release hormones into the hepatic venous circulation, and these active hormones enter the systemic circulation before the hepatic metabolism, leading to hormonal excess syndromes. Liver-directed therapies, including surgery, have a significant impact on hormonal excess syndromes induced by NET LMs. Complete resection of NET LMs leads to prolonged survival: 5 -year overall survival rates $>70 \%$ $[7,8]$ are reported despite recurrence in up to $80 \%$ of patients [9]. Thus the rationale to resect NET LMs when feasible is established.

\section{Pre-treatment evaluation and diagnostic imaging}

Pre-treatment staging often includes the biochemical assessment of serologic markers such as serotonin, insulin, vasoactive intestinal peptide (VIP), and chromogranin, even in patients without clinically apparent hormonal excess. When one or more of these serum markers are elevated, they can be used to assess the efficacy of treatment and may be helpful in the diagnosis of recurrence after therapy. Patients with hormonal excess (e.g. flushing, diarrhoea, bronchospasm, hypoglycaemia or carcinoid heart disease as a result of tricuspid insufficiency) may lack the cardiovascular or nutritional reserve required for safe major surgery. This subset of patients may require treatment of the effects of hormone excess using somatostatin analogues, anti-diarrhoeal or other therapies in order to improve performance status for more definitive treatment. Occasionally, other non-operative interventions, such as tumour embolization or chemotherapy, can be considered (these are discussed below), and surgery is considered once performance status improves. Echocardiography is indicated when cardiac function is impaired, and severe valve dysfunction prompts repair before consideration for hepatic surgery [10]. In patients with carcinoid heart disease, hepatic resection is associated with decreased risk of cardiac progression and improved 5-year survival [11]. Staging in patients being considered for hepatic metastasectomy includes whole-body imaging, and liver-specific, anatomical imaging. Whole body functional studies which can be helpful to assess and characterize disease extent include Tc99m-HYNIC-TOC scintigraphy, or newer techniques such as gallium-68 DOTA-TATE PET/CT. 9-fluorodeoxyglucose positron emission tomography (FDG-PET) may be considered for staging high grade NETs. Radiolabelled octreotide has high affinity for somatostatin receptor 2 (sst2), thereby enabling specific binding to NETs, whereas FDG-PET relies on glucose consumption to highlight metabolically active tumours. The sensitivity of octreotide scanning for patients with GEP-NETs is 89-90\%, and may approach $100 \%$ with glucagonoma, nearly $75 \%$ for gastrinoma, $85 \%$ for carcinoid, and about $90 \%$ for VIPoma $[12,13]$. Detection of octreotide avid lymph nodes and liver lesions is virtually pathognomonic for metastatic NETs and can guide planned surgical therapy. PET should be fused with computed tomography (CT) imaging to provide relative anatomic information regarding tumour location, and as such can significantly improve the utility of these whole-body functional tests in surgical planning. Other findings on CT imaging or magnetic resonance imaging $(\mathrm{MRI})$ can also significantly impact surgical planning, such as anatomic variations or vascular issues. The objectives of staging with cross-sectional imaging using MRI or CT are 3-fold: firstly, to assess the distribution of tumours throughout the liver, including millimetric capsular disease if possible; secondly, to define the intrahepatic vascular anatomy and the association of tumours with intrahepatic vascular structures; and finally, when extensive resection is required, to enable 3-dimensional liver volumetry of the FLR.

\section{Assessment of performance status and clinical assessment of liver function}

In the context of a potential surgical resection of NET LMs, the performance status and the liver function are evaluated. In cases of severe hormonal syndrome or significant hepatic involvement, patient performance status may be poor. In surgical candidates, liver function after hepatectomy is contingent on the quality of the liver parenchyma, the volume of the FLR, and the regenerative capacities of the liver. The risk of postoperative liver failure remains one of the main concerns after major liver resection. This risk must be estimated preoperatively not only to determine the safety of resection but also because preoperative steps can often be taken to avoid 
postoperative liver dysfunction. Patients with NET LMs who are candidates for major liver resection generally have normal liver function. For the most part, aside from steatosis associated with somatostatin analogues and perhaps streptozocin, severe hepatic parenchymal injuries are not commonly a major problem in candidates for resection of NET LMs [14]. Thorough preoperative evaluation should detect portal hypertension that would increase the risk associated with surgery. Careful clinical examination to exclude liver disease (ascites, collateral venous circulation), biologic assessment (for platelet count < 100 000) and imaging (for evidence of venous collaterals, oesophageal varices, morphologic changes in liver shape consistent with chronic liver disease, and splenomegaly) allow the selection of patients suitable for major liver resection. When imaging suggests severe underlying liver disease, laparoscopy with or without liver biopsy of the non-tumourous liver parenchyma can aid in quantifying underlying disease, but its routine use is generally not necessary. Evaluation of liver disease is critical to determine the safe extent of liver resection.

\section{Assessment of the FLR}

Liver volume and liver function are linked [15]. In fact, there is a direct correlation of liver volume to patient size: large patients have large livers, smaller patients smaller livers. Thus, when extensive liver resection is planned, assessment of the FLR volume is indicated in order to determine the safety of resection and determine whether preoperative liver enhancement is needed, because the disease-free liver that remains (not the tumour-bearing liver that is removed) determines the safety of resection, recovery and postoperative liver function of the individual patient. The importance of focus on the FLR prior to resection relates to the absence of effective treatment for postoperative severe hepatic failure related to an inadequate remnant [16], and to the existence of preoperative preparatory treatments (specifically PVE), which can reduce the risk both of fatal hepatic failure but also hepatic insufficiency, which can lead to prolonged, complicated postoperative recovery. The limits of safe, extensive liver resection are gradually being refined. Liver function is affected by the presence and degree of underlying liver disease: patients with abnormal underlying liver parenchyma require larger FLR volumes following resection to ensure adequate post-resection liver function [17]. Rigorous data have defined safe limits for resection, and agreement on these limits has been reached in an international consensus based on extensive data. Specifically, for patients with normal underlying liver, the FLR volume limit based on standardized FLR volumetry is $20 \%$ of total liver volume (TLV) $[18,19]$. Patients with significant underlying liver disease require $30 \%$ of TLV [20]. For patients with well-compensated cirrhosis, $40 \%$ of TLV is required for safe major hepatectomy [21, 22]. When patients are considered for major hepatectomy, volumetry of the FLR is indicated.

\section{Surgical outcomes: liver resection for NET LMs}

The frequency of LMs may be greater than $40 \%$ in patients with NETs depending on the primary site. Medical therapy alone tends to be marginally effective, with 5 -year survival ranging from 0 to $30 \%$. The relatively indolent nature of this disease and its tendency to be hormonally active merit aggressive therapy even for advanced disease. Aggressive surgical management with curative intent has been demonstrated to improve 5-year survival in many series with acceptable morbidity and mortality [23]. Even aggressive concomitant resection of NET LMs with the primary NET has been advocated by some to be warranted, based on an overall 5 -year survival of $71 \%$ [24]. However, recurrence rates even with aggressive surgical management have been demonstrated to be as high as $84 \%$. One of the largest series of patients undergoing hepatic resection for metastatic neuroendocrine disease has been reported by investigators working at the Mayo Clinic, who examined 170 surgically treated patients and demonstrated a surgical morbidity of $14 \%$ and mortality of $1.2 \%$. Recurrence developed in $84 \%$ of patients, and the 5 -year overall survival was $61 \%$ with a median survival of 81 months. There was no difference in overall among patients with preoperative hormonal symptoms compared with patients without endocrinopathies. A recent study of 172 patients undergoing procedures for NET LMs demonstrated a morbidity rate of $22 \%$ with a mortality rate of $0 \%$ in the $140(81.4 \%)$ patients who underwent hepatic surgery. Eighty (46.5\%) patients developed recurrence of LMs and underwent either re-resection or ablative therapy. The median overall survival was 9.64 years with a 5 -year overall survival rate of $77.4 \%$. A multi-institutional study identified 339 patients who underwent surgical intervention (79\% surgical resection, $3 \%$ ablation, $19 \%$ surgery and ablation, as initial liver-directed procedures) over a 14-year period. Median overall survival was 125 months with 5- and 10-year overall survival of $71 \%$ and $51 \%$, respectively [25]. Liver resection may be indicated to improve a patient's quality of life and/or decrease the need for medical therapy where performance status permits consideration for surgery.

\section{Radiofrequency ablation}

Radiofrequency ablation (RFA) is a technique that involves the use of thermal energy to induce coagulation 
necrosis, thereby destroying tumour cells. When heated to $45-50^{\circ} \mathrm{C}$, proteins are denatured and cellular membranes are destroyed. Utilizing an alternating electric current in the range of radiofrequency waves applied via needle electrodes directly into the tumour, localized thermal ablation with temperatures of greater than $60^{\circ} \mathrm{C}$ may be achieved [26]. Although surgical resection remains the mainstay of treatment for NET LMs, RFA may serve as either a primary modality to treat LMs or an adjunct to surgery in many instances. Ablative therapy, for the most part, is associated with low morbidity and mortality while providing a satisfactory level of symptomatic control despite a near universal local recurrence rate for treated patients [27]. One of the earlier studies of RFA for NET LMs included a series of 34 patients with 234 LMs who underwent a total of 42 ablative sessions. Morbidity was minimal at $5 \%$ with no reported periprocedural mortality. Mean survival after RFA was only 1.6 years with new liver lesions in $28 \%$ and local recurrence in $13 \%$ [28], but amelioration of symptoms was achieved in $95 \%$ of patients with significant relief in $16 \%$ and complete resolution of symptoms in $63 \%$, suggesting some clinical value to the ablative approach in these selected patients. In a retrospective analysis of RFA done for NET LMs, 80 laparoscopic RFA sessions were performed in 63 patients over a 10-year period. Perioperative morbidity was minimal at $5 \%$ and there was no perioperative mortality. Ablation afforded partial symptomatic relief in $92 \%$ and complete relief in $70 \%$ patients. Median survival was 3.9 years after the first session of RFA with a local recurrence rate of $6.3 \%$ [29]. In the study mentioned above, which analysed 339 patients undergoing liver-directed therapies for metastatic NETs, the 5 -tear overall survival was $72 \%$ while progression-free survival (PFS) was $4.5 \%$ in the subset of patients who underwent ablation [30]. Thus RFA is clearly a useful tool for the treatment of NET LMs. Limitations of the technique include the poor efficacy of ablation in tumours $>3-4 \mathrm{~cm}$ in diameter, and a contraindication for ablation $<1 \mathrm{~cm}$ from central biliary or portal venous structures (as the RFA heat can lead to biloma, portal thrombosis and death). RFA is extremely useful for the treatment of small tumours, and is very effectively used in patients after resection.

\section{Resection plus RFA}

Resection plus RFA is increasingly used in patients with bilateral NET LMs. Resection is performed for large or dominant lesions, while ablation is used to treat small lesions in the FLR. Usually, both resection and ablation are performed simultaneously at laparotomy. Resection plus RFA has been used to treat metastatic colorectal and non-colorectal disease. In a series of
172 patients treated by the combined modalities, the morbidity rate was $19.8 \%$ with a mortality rate of $2.3 \%$, comparable to much of the data for resection alone. The recurrence rate was $56.9 \%$ at median follow-up of 21.3 months [31]. Liver failure was attributed to the degree of thermal destruction caused by the ablative therapy in the small FLR, bringing to light a potential risk associated with this one-stage approach to bilateral tumours. Therefore, great care must be undertaken to select appropriate patients for combined therapy, particularly in those individuals with underlying compromised liver function. This disadvantage of resection plus RFA necessitates the formal assessment of FLR function: this approach should be reserved for patients with a definitively adequate FLR who require ablation of relatively small metastatic lesions in the FLR not associated with the major vascular structures therein. Certainly if resection plus RFA is planned and there is concern that ablation may damage the FLR, observation and reoperation to treat residual disease (or postoperative percutaneous therapy) might be considered to ensure a safe outcome.

\section{Liver transplantation}

Liver transplantation (LT) may be considered in a highly selected group of patients with NET LMs. In contrast to LT outcomes performed for other secondary liver malignancies, the outcomes of LT for fairly extensive but liver-limited NET LMs have been promising. LT is generally considered when resectable (usually resected) primary and locoregional disease without extrahepatic metastases or unresectable hepatic metastatic disease is present (with or without hormonal symptoms refractory to medical management). Often, progressive hepatic failure related to hepatic tumour burden is present, and patients are otherwise not candidates for liver directed therapies [32]. Van Vilsteren defined selection criteria for LT in NETs as follows: (1) histological confirmation of NET LMs or a primary NET with evidence of LMs, (2) bilobar, unresectable liver metastasis, (3) complete resection of the primary NET, (4) the absence of extrahepatic disease, and (5) the patient must be deemed a suitable candidate by a transplant team and is on a transplant list [33]. These or similar guidelines are used in most countries. However, a French multicentre study of 85 patients transplanted for metastatic NETs described factors which might enhance LT selection for NETs [34]. Firstly, a 47\% 5-year overall survival was reported for the entire registry cohort. Secondly, they identified primary tumours in the duodenum or pancreas, as well as upper abdominal exenteration, as predictors of poor survival, and reported that 5-year survival reached $68 \%$ among 55 patients with one or neither poor prognostic factor. These findings have 
been perceived as potential factors which could allow for better selection of patients with NET LMs for LT, with an expectation for $>50 \% 5$-year overall survival and excellent quality of life in properly selected patients. More work is needed, but selection criteria may exclude those with the need for upper abdominal exenteration, patients with pancreatic NETs, and patients with hepatomegaly, as these three groups may not derive sufficient benefit from LT based on this literature review [35]. Other factors which may contribute to NET patient selection for LT may be gleaned from other studies on this topic, which reinforce the potential for a good outcome with this therapy despite relatively advanced disease. 47-51\% survival rates are reported in larger studies [36, 37]; these studies also suggest that favourable prognostic factors include age less than 50 years, primary tumour located in the lung or bowel, and preoperative somatostatin therapy. Several molecular markers have been investigated as prognostic indicators to refine the selection process. Most notably, the expression of Ki67, a nuclear protein located in proliferating cells, has been found to be inversely correlated to survival outcomes, although this finding has not been supported by all studies. A meta-analysis of 4 series examined the use of the Ki67 labelling index as a prognostic indicator [38-40]. Seventy-seven patients were combined from all 4 series and divided into a low-risk group (Ki67 index $<2 \%$ ) and a high-risk group (Ki67 index > 2\%). Low-risk patients demonstrated improved 1- and 3-year survival and recurrence-free survival when compared to highrisk patients. Moreover, recurrence was $52 \%$ at 3 years for the low-risk group compared to $17 \%$ in the highrisk group [41]. The improvement in imaging modalities, especially in functional imaging with 68Ga DOTA-TATE $\mathrm{PET} / \mathrm{CT}$, enables more robust assessment of patients to exclude extra-hepatic disease, and thus better outcomes in future studies can be expected.

\section{Transarterial liver directed therapies}

Less than $10 \%$ of patients with metastatic NETs of the liver are candidates for surgical resection. Systemic chemotherapy has limited efficacy in GEP-NETs and generates more robust, albeit variable, responses in pancreatic NETs. Hepatic arterial embolization, typically termed transarterial embolization (TAE), and transarterial chemoembolization (TACE) have been shown to induce reduction in tumour size and to ameliorate symptoms of excess hormonal secretion. The rationale for use of liver directed therapies is predicated on the observation that metastatic liver lesions derive much of their nutrient supply from the hepatic arteries. In a series of 69 patients with metastatic carcinoid tumours and 54 patients with metastatic pancreatic NETs who underwent TAE or TACE, a higher response rate, longer PFS, and longer overall survival were found in those with carcinoid tumours. For patients with carcinoid tumours, TAE generated a higher response than TACE $(p=0.004)$ and lower toxicity. For patients with pancreatic NETs, TACE resulted in an improved response rate and overall survival, though statistical significance was not achieved [42]. Extensive experience with TAE and TACE for carcinoid and other NETs has secured the place of these approaches for treatment of LMs. The results of the major studies that examined the utility of TAE and TACE for NETs reveal 33-74\% response rates, though more recent studies quite consistently demonstrate radiographic response rates approximately $50 \%$ or higher $[43,44]$. The few studies that report symptom response suggest much higher rates of symptom response, ranging from 70 to $100 \%$. Emerging data regarding the use of radioactive microspheres for embolization, known as radioembolization, suggest that the technique has some utility for the treatment of metastatic NETs. Yttrium-90 ( $\left.{ }^{90} \mathrm{Y}\right)$ microspheres are available in two forms, one with ${ }^{90} \mathrm{Y}$ glass beads which have a dual effect of delivery of radioactive material to the tumours and generating an arterial embolic effect, and a second form with ${ }^{90} \mathrm{Y}$ labelled resin microspheres, which deliver a lower dose of radiation and lack a significant embolic effect $[45,46]$. This report is not designed to detail the differences between the two radioembolization techniques, but it is worth noting that different radioactive particle delivery systems are just that, different, and cannot be viewed interchangeably. TAE can be an extremely useful adjunct to liver resection in some patients. The classic example is a patient with severe hormonal excess leading to nutritional compromise, yet whose metastatic disease in the liver is resectable. Such patients may derive significant benefit from preoperative therapy with TAE. If the hormonal excess syndrome is controlled, subsequent definitive resection can provide long-term, symptom-free survival. The combination of careful attention to preoperative patient preparation, nonsurgical and surgical therapy, and meticulous surgical technique optimizes outcomes by taking advantage of the many aspects of multidisciplinary care.

\section{Systemic therapy}

While systemic chemotherapy can be of tremendous value in patients with well- and moderately differentiated pancreatic NETs, these and other agents have little effect in small bowel carcinoids. Chemotherapy options may consist of monotherapy or a combination of the following agents: temozolomide, capecitabine, streptozocin, 5-fluorouracil, doxorubicin, dacarbazine, platinum-based compounds (carboplatin, cisplatin, oxalipla- 
tin) or etoposide. Poorly differentiated NETs regardless of origin are generally more responsive to platinumbased therapy, occasionally irinotecan and etoposide [47]. The choice of systemic chemotherapy in the management of pancreatic NETs is an area of debate because of reports suggesting a wide range of response from $6 \%$ to $69 \%$ for streptozocin-based chemotherapy. Octreotide analogues have gained a secure place in the treatment of patients with metastatic NETs since the publication of the PROMID study (Placebo-controlled prospective Randomized study on the antiproliferative efficacy of Octreotide LAR in patients with metastatic MIDgut NETs) which demonstrated the ability of the long-acting Sandostatin analogue octreotide LAR to delay progression in treated patients [48]. This quality (providing stability) has a different effect than the decreased tumour size associated with cytotoxic therapy for pancreatic NETs, but is also of value for the surgeon. Announced results of the CLARINET study, which concerned the use of lanreotide autogel in GEP-NEN, confirmed the antiproliferative effect of this analogue. The study involved 204 patients with NEN G1 and G2 (Ki67 < 10\%) inactive with primary pancreas (45\%), midgut (36\%), hindgut (7\%) and unknown (13\%) and liver involvement > 25\% in 33\% of patients. Two-year lanreotide treatment with $120 \mathrm{mg}$ autogel every 4 weeks showed no disease progression or death in $62 \%$ of patients compared to $22 \%$ of patients receiving placebo [49]. Among patients with a significant disease burden but who might be candidates for treatment, observation on octreotide and lanreotide may provide longitudinal data in the specific patient, and may help reveal the biological behaviour of that patient's tumour. Accordingly, and in concert with a seasoned medical oncologist, the patient can be selected for aggressive surgical approaches when there is a clear picture of the biology of the individual patient's tumour.

\section{Conclusions}

In patients with NET LMs, the small intestine and pancreas are the most common primary tumour sites. Many patients will have prolonged survival even in the context of advanced metastatic disease; therefore aggressive treatment strategies must be applied with care. No single treatment approach or algorithm can be designed for patients with NET LMs. Although a cure is elusive, complete resection offers a significant probability for long-term survival in patients with NET LMs: 5 -year survival rates of $70 \%$ or more are consistently reported following resection [50-52]. Incomplete resection or debulking has been described as a strategy to be considered in highly selected cases to palliative severe hormonal syndrome [53], though most experienced surgeons and centres are reluctant to pursue debulking because of the apparently small advantage in survival beyond an expected 30\% 5-year survival rate among non-surgically managed patients. Management of patients with NETs metastatic to the liver requires an integrated, multidisciplinary approach. Patients with hormonal excess may suffer nutritional compromise that precludes consideration for up-front surgery, and sequential medical and surgical therapy may be needed. Fortunately, a growing proportion of patients with functional and nonfunctional NET LMs can be considered for complete resection of LMs, even when a significant tumour burden or bilateral disease is present, because of advances in surgical techniques, and combination of medical and surgical therapy. A proportion of patients can benefit from a combination of therapies that may address the disease process in a locoregional or systemic manner even if the intent is not for cure. Locoregional therapies can be used to control hormonal symptoms in preparation for surgery. Percutaneous or transarterial approaches may be used alone or in combination with surgery. Systemic therapies include biotherapeutic agents and traditional cytotoxic agents; somatostatin analogues have demonstrated effectiveness in symptomatic control and may afford some level of tumour response. Many patients, not otherwise considered candidates for surgery, benefit from a sequence of medical and interventional therapies which may open doors to more durable surgical interventions. Simultaneously, indications for extensive liver resection are expanding in general, and specifically for patients with NET LMs. With safe extended resection and two-stage surgical approaches to patients with bilateral liver tumours, a growing population of patients with NET LMs is considered for surgery. Ablative therapies have an established adjunctive role in disease control in highly selected patients with NET LMs. Liver transplantation can be considered when extrahepatic disease is eradicated and in rare cases of overwhelming liver disease. Essential to a good outcome is the continuous interaction between members of the multidisciplinary team to optimally sequence treatments to enable the best oncologic and clinical outcomes for patients with this challenging family of diseases.

\section{Conflict of interest}

The author declares no conflict of interest.

\section{References}

1. McDermott EW, Guduric B, Brennan MF. Prognostic variables in patients with gastrointestinal carcinoid tumours. Br J Surg 1994; 81: 1007-9.

2. Norton JA, Kivlen M, Li M, et al. Morbidity and mortality of aggressive resection in patients with advances neuroendocrine tumors. Arch Surg 2003; 138: 859-66. 
3. Godwin JD. Carcinoid tumors. An analysis of 2,837 cases. Cancer 1975; 36: 560-9.

4. Zeitels J, Naunheim K, Kaplan EL, et al. Carcinoid tumors: a 37year experience. Arch Surg 1982; 117: 732-7.

5. Jensen RT. Natural history of digestive endocrine tumours. In: Recent Advances in the Pathophysiology and Management of Inflammatory Bowel Disease and Digestive Endocrine Tumours. Colombel JF, Mignon M (eds.). John Libbey Eurotext, Paris, France 1999; 192-219.

6. Van Gompel JJ, Sippel RS, Warner TF, et al. Gastrointestinal carcinoid tumors: factors that predict outcome. World J Surg 2004; 28: 387-92.

7. Elias D, Cavalcanti de Albuquerque A, Eggenspieler P, et al. Resection of liver metastases from a noncolorectal primary: indications and results based on 147 monocentric patients. J Am Coll Surg 1998; 187: 487-93.

8. Glazer ES, Tseng JF, Al-Refaie W, et al. Long-term survival after surgical management of neuroendocrine hepatic metastases. HPB (Oxford) 2010; 12: 427-33.

9. Sarmiento JM, Heywood G, Rubin J, et al. Surgical treatment of neuroendocrine metastases to the liver: a plea for resection to increase survival. J AM Coll Surg 2003; 197: 29-37.

10. McDonald ML, Nagorney DM, Connolly HM, et al. Carcinoid heart disease and carcinoid syndrome: successful surgical treatment. Ann Thorac Surg 1999; 67: 537-9.

11. Bernheim AM, Connolly HM, Rubin J, et al. Role of hepatic resection for patients with carcinoid heart disease. Mayo Clin Proc 2008; 83: 143-50.

12. Krenning EP, Kwekkeboom DJ, Oei HY, et al. Somatostatin receptor scintigraphy in carcinoids, gastrinomas and Cushing s syndrome. Digestion 1994; 55 (Suppl 3): 54-9.

13. Kwekkeboom DJ, Krenning EP. Somatostatin receptor imaging Semin Nucl Med 2002; 32: 84-91.

14. Attanasio R, Mainolfi A, Grimaldi F, et al. Somatostatin analogs and gallstones: a retrospective suevey on a large series of acromegalic patients. J Endocrinol Invest 2008; 31: 704-10.

15. Abdalla EK. Portal vein embolization (prior to major hepatectomy) effects on regeneration, resectability, and outcome. J Surg Oncol 2010; 102: 960-7.

16. Abdalla EK, Hicks ME, Vauthey JN. Portal vein embolization: rationale, technique and future prospects. Br J Surg 2001; 88: 165-75.

17. Abdalla EK, Adam R, Bilchik AJ, et al. Improving resectability of hepatic colorectal metastases: expert consensus statement. Ann Surg Oncol 2006; 13. 1271-80.

18. Kishi Y, Abdalla EK, Chun YS, et al. Three hundred and one consecutive extended right hepatectomies: evaluation of outcome based on systematic liver volumetry. Ann Surg 2009; 250: 540-8.

19. Ribero D, Curley SA, Imamura $\mathrm{H}$, et al. Selection for resection of hepatocellular carcinoma and surgical strategy: indications for resection, evaluation of liver function, portal vein embolization, and resection. Ann Surg Oncol 2008; 15: 986-92.

20. Vauthey JN, Dixon E, Abdalla EK, et al. Pretreatment assessment of hepatocellular carcinoma: expert consensus statement. HPB 2010; 12: 289-99.

21. Kubota K, Makuuchi M, Kusaka K, et al. Measurement of liver volume and hepatic functional reserve as a guide to deci- sion-making in resectional surgery for hepatic tumors. Hepatology 1997; 26: 1176-81.

22. Shirabe K, Shimada M, Gion T, et al. Postoperative liver failure after major hepatic resection for hepatocellular carcinoma in the modern era with special reference to remnant liver volume. J Am Coll Surg 1999; 188: 304-9.

23. Musunuru S, Chen H, Rajpal S, et al. Metastatic neuroendocrine hepatic tumors: resection improves survival. Arch Surg 2006; 141: 1000-4.

24. Sarmiento JM, Que FG, Grant CS, et al. Concurrent resections of pancreatic islet cell cancers with synchronous hepatic metastases: outcomes of an aggressive approach. Surgery 2002; 132: $976-82$.

25. Mayo SC, de Jong MC, Pulitano C, et al. Surgical management of hepatic neuroendocrine tumor metastasis: results from an international multi-institutional analysis. Ann Surg Oncol 2010; 13: 2141-51.

26. Curley SA. Radiofrequency ablation of malignant liver tumors. Oncologist 2001; 6: 14-23.

27. Atwell TD, Charboneau JW, Que FG, et al. Treatment of neuroendocrine cancer metastatic to the liver: the role of ablative techniques. Cardiovasc Intervent Radiol 2005; 28: 409-21.

28. Berber E, Flesher N, Siperstein AE. Laparoscopic radiofrequency ablation of neuroendocrine liver metastases. World J Surg 2002; 26: 985-90.

29. Mazzaglia PJ, Berber E, Milas M, et al. Laparoscopic radiofrequency ablation of neuroendocrine liver metastases: a 10-year experience evaluating predictors of survival. Surgery 2007; 142: 10-9.

30. Pawlik TM, Izzo F, Cohen DS, et al. Combined resection and radiofrequency ablation for advanced hepatic malignancies: results in 172 patients. Ann Surg Oncol 2003; 10: 1059-69.

31. Kianmanesh R, Farges O, Abdalla EK, et al. Right portal vein ligation: a new planned two-step all-surgical approach for complete resection of primary gastrointestinal tumors with multiple bilateral liver metastases. J Am Coll Surg 2003; 197: 164-70.

32. Frilling A, Li J, Malamutmann E, et al. Treatment of liver metastases from neuroendocrine tumours in relation to the extent of hepatic disease. Br J Surg 2009; 96: 175-84.

33. van Vilsteren FG, Baskin-Bey ES, Nagorney DM, et al. Liver transplantation for gastroenteropancreatic neuroendocrine cancers: defining selection criteria to improve survival. Liver Transpl 2006; 12: 448-56.

34. Le Treut YP, Gregoire E, Belghiti J, et al. Predictors of long-term survival after liver transplantation for metastatic endocrine tumors: an 85-case French multicentric report. Am J Transplant 2008; 8: 1205-13.

35. Gregoire E, Le Treut YP. Liver transplantation for primary or secondary endocrine tumors. Transpl Int 2010; 23: 704-11.

36. Lehnert T. Liver transplantation for metastatic neuroendocrine carcinoma: an analysis of 103 patients. Transplantation 1998; 66: 1307-12.

37. Que FG, Sarmiento JM, Nagorney DM. Hepatic surgery for metastatic gastrointestinal neuroendocrine tumors. Adv Exp Med Biol 2006; 574: 43-56.

38. Ahlman H, Friman S, Cahlin C, et al. Liver transplantation for treatment of metastatic neuroendocrine tumors. Ann N Y Acad Sci 2004; 1014: 265-9. 
39. Olausson M, Friman S, Herlenius G, et al. Orthotopic liver or multivisceral transplantation as treatment of metastatic neuroendocrine tumors. Liver Transpl 2007; 13: 327-33.

40. Rosenau J, Bahr MJ, von Wasilewski R, et al. Ki 67, E-cadherin, and p53 as prognostic indicators of long-term outcome after liver transplantation for metastatic neuroendocrine tumors. Transplantation 2002; 73: 386-94.

41. Grossman EJ, Millis JM. Liver transplantation for non-hepatocellular carcinoma malignancy: Indications, limitations, and analysis of the current literature. Liver Transpl 2010; 16: 930-42.

42. Gupta S, Johnson MM, Murthy R, et al. Hepatic arterial embolization and chemoembolization for the treatment of patients with metastatic neuroendocrine tumors: variables affecting response rates and survival. Cancer 2005; 104: 1590-602.

43. Nazario J, Gupta S. Transarterial liver-directed therapies of neuroendocrine hepatic metastases. Semin Onkol 2010; 37: 118-26.

44. Strosberg JR, Cheema A, Kvols LK. A review of systemic and liver-directed therapies for metastatic neuroendocrine tumors of the gastroenteropancreatic tract. Cancer Control 2011; 18: 127-37.

45. Kennedy AS, Dezarn WA, McNeillie P, et al. Radioembolization for unresectable neuroendocrine hepatic metastases using resin 90Y-microspheres: early results in 148 patients. Am J Clin Oncol 2008; 31: 271-9.

46. King J, Quinn R, Glenn DM, et al. Radioembolization with selective internal radiation microspheres for neuroendocrine liver metastases. Cancer 2008; 113: 921-9.

47. Yalcin S. Advances in the systemic treatment of pancreatic neuroendocrine tumors. Cancer Treat Rev 2010; 37: 127-32.

48. Rinke A, Muller HH, Schade-Brittinger C, et al. Placebo-controlled, double-blind, prospective, randomized study on the effect of octreotide LAR in the control of tumor growth in patients with metastatic neuroendocrine midgut tumors: a report from the PROMID Study Group. J Clin Oncol 2009; 27: 4656-63.

49. Caplin ME, Pavel M, Ćwikła JB, et al.; CLARINET Investigators. Anti-tumour effects of lanreotide for pancreatic and intestinal neuroendocrine tumours: the CLARINET open-label extension study. Endocr Relat Cancer 2016; 23: 191-9.

50. Adam R, Chiche L, Aloia T, et al. Hepatic resection for noncolorectal nonendocrine liver metastases: analysis of 1,452 patients and development of a prognostic model. Ann Surg 2006; 244: 524-35.

51. Choi EA, Abdalla EK. Patient selection and outcome of hepatectomy for noncolorectal nonneuroendocrine liver metastases. Surg Oncol Clin N Am 2007; 16: 557-77.

52. Ercolani G, Grazi GL, Ravaioli M, et al. The role of liver resections for noncolorectal, nonneuroendocrine metastases: experience with 142 observed cases. Ann Surg Oncol 2005; 12: 459-66.

53. Weitz J, Blumgart LH, Fong Y, et al. Partial hepatectomy for metastases from noncolorectal, nonneuroendocrine carcinoma. Ann Surg 2005; 24: 269-76.

Received: 24.10.2019

Accepted: 30.11 .2019 